\title{
Allelic methylation status of CpG islands on chromosome 21q in patients with Trisomy 21
}

\author{
YIN-YIN XIA ${ }^{1}$, YU-BING DING ${ }^{2}$, XUE-QING LIU ${ }^{2}$, XUE-MEI CHEN ${ }^{2}$, SHU-QUN CHENG $^{1}$, \\ LIAN-BING LI ${ }^{3}$, MING-FU MA ${ }^{3}$, JUN-LIN $\mathrm{HE}^{2}$ and YING-XIONG WANG ${ }^{2}$
}

\begin{abstract}
Departments of ${ }^{1}$ Occupational and Environmental Hygiene and ${ }^{2}$ Reproductive Biology, Chongqing Medical University;
${ }^{3}$ Chongqing Key Laboratory of Birth Defects and Reproductive Health, Institute for Science and Technology Research of Chongqing Population and Family Planning, Chongqing 400016, P.R. China
\end{abstract}

Received May 23, 2013; Accepted January 30, 2014

DOI: $10.3892 / \mathrm{mmr} .2014 .1985$

\begin{abstract}
Trisomy 21 is a chromosomal condition caused by the presence of all or part of an extra 21st chromosome. There has been limited research into the DNA methylation status of $\mathrm{CpG}$ islands (CGIs) in trisomy 21, therefore, exploring the DNA methylation status of CGIs in $21 \mathrm{q}$ is essential for the development of a series of potential epigenetic biomarkers for prenatal screening of trisomy 21. First, DNA sequences of CGIs in 21q from the USCS database were obtained and 149 sequences and 148 pairs of primers in the BGI YH database were aligned. All 300 cases were analyzed by a heavy methyl-polymerase chain reaction (HM-PCR) assay and a comparison of the DNA methylation status of CGIs was made between trisomy 21 and the control. The HM-PCR assay results did not show a difference in the DNA methylation status between individuals with trisomy 21 and the control. In total, there were 11 CGIs that showed various DNA methylation statuses between Japanese and Chinese patients. Subsequently, bisulfite genomic sequencing found variations in the methylation status of CpG dinucleotides in CGIs (nos. 14, 75, 109, 134 and 146) between trisomy 21 and the control. The different DNA methylation status of CpG dinucleotides in CGIs may be a potential epigenetic marker for diagnosing trisomy 21 . No difference was identified in the DNA methylation status of 21q CGIs among Chinese individuals with trisomy 21 and the control. The homogeneity of the DNA methylation status of 21q CGIs in Chinese patients indicates that DNA methylation is likely to be an epigenetic marker distinguishing ethnicities.
\end{abstract}

Correspondence to: Dr Jun-Lin He or Dr Ying-Xiong Wang, Department of Reproductive Biology, Chongqing Medical University, East Building, No. 1 Yixueyuan Road, Chongqing 400016, P.R. China

E-mail: 1754399492@qq.com

E-mail: 1600347366@qq.com

Key words: CpG islands, trisomy 21, DNA methylation, chromosome pair 21, epigenetic marker

\section{Introduction}

Down's syndrome (DS) or trisomy 21 is a chromosomal condition caused by the presence of all or part of an extra 21st chromosome. DS is a high-incidence birth defect that is often associated with impairment of cognitive ability and physical growth (1). Individuals with DS have a higher risk of congenital heart disease (CHD), dysfunction of the thyroid gland, Hirschsprung's disease, eye and hearing disorders, leukemia and testicular cancer; however, there is a wide range of phenotypic variation in DS (2-6). It is now more than half a century since DS was first shown to result from trisomy 21 (7). Although progress has been made by investigating genes to understand the complex phenotypes associated, the mechanisms remain far from clear.

DS disorders are the result of extra copies of the genes located on chromosome 21. In general, an overexpression of the genes arises and DNA hypomethylation is a possible mechanism to explain the altered gene expression. DNA methylation often occurs in a $\mathrm{CpG}$ dinucleotide, in which the cytosine gains a methyl group. Hypermethylation results in transcriptional silencing, for example genomic imprinting and $\mathrm{X}$-chromosome inactivation, while hypomethylation is linked to chromosomal instability and loss of imprinting. Unmethylated CpGs are often grouped in CpG islands (CGIs), which are present in the 5 ' regulatory regions of a number of genes and acquire abnormal hypermethylation in numerous disease processes. Alterations of DNA methylation have been recognized as an important component of cancer development (8). Altered methylation status in peripheral blood lymphocytes (PBLs) has been linked to increased risk of several diseases and, in addition, PBLs are an easily accessible source to identify potential epigenetic biomarkers (9).

In the present study, a comprehensive CGI methylation analysis was performed using trisomy 21 and control cases to identify the allelic methylation status in CGIs of PBLs. The aim of the study was to determine the utility of CGI methylation analysis associated with human diseases, in particular, the DS complex phenotypes and to locate potential epigenetic biomarkers for prenatal diagnosis. To the best of our knowledge, there are no comprehensive CGI methylation analyses that have focused on chromosome $21 \mathrm{q}$ using a large number 
of trisomy 21 samples (10), thus, the present study focused on chromosome 21, which is the major contributor of DS complex phenotype.

\section{Materials and methods}

Alignment of CGI data to the BGI YH database. The present analysis was based on the results of a comprehensive measurement of CGI methylation on human chromosome 21q (11). Yamada et al repeat-masked the chromosome sequence and computationally identified all non-repetitive CGIs using standard tools and parameters (GC content, $>50 \%$; ratio of observed versus expected number of $\mathrm{CpG}$ dinucleotides, $>0.6$; $>400$ base pairs in length). The authors designed primers for the 149 CGI identified and extracted corresponding DNA from samples of human PBLs. Finally, the authors determined the methylation status of each CGI using methylation-specific restriction enzymes (via HpaII-McrBC-PCR). The DNA sequences of 149 CGIs were acquired from UCSC (11) and were aligned with the sequence in BLAST, the BGI YH database (http://yh.genomics.org.cn/search.jsp). The score of each alignment was indicated by one of five colors, in which the highest score was $>200$ and shown in red. Multiple alignments on the same database sequence were connected by a striped line. A continuous red line indicated a perfect match (100\% match) and the alignment data was classified into ten groups based upon the percentage of red line. Subsequently, the primers of the 148 CGIs (11) were aligned in sequence in the BLAST BGI YH database (CGI no. 103 was investigated with bisulfite sequencing as it lacked $\mathrm{HpaII}$ and $\mathrm{HhaI}$ sites and could not detected by HM-PCR). When a base differed to a sequence in the database, the primer was a $0 \%$ match. The alignment results were divided into three groups: Perfect match (a pair of primers was 100\% matched); $50 \%$ match (one primer of a pair was $100 \%$ matched); and no match (a pair of primers was $0 \%$ matched).

Study subjects and diagnosis. A total of 150 control cases and 150 DS cases were obtained through the Children's Hospital of Chongqing Medical University (Chongqing, China) and participant's families or correspondents provided informed consent. The distribution of age and residential placement did not differ between the control and the patients. Confirmation of trisomy 21 was obtained by G-banded karyotypes, all patients had complete trisomy 21 , with $100 \%$ concordance between cytogenetics and the clinical diagnosis of DS. Data for Japanese individuals were obtained from the study by Yamada et al (11).

Preparation of genomic DNA from human PBLs. Trisomy 21 and normal human lymphocytes were prepared from peripheral blood. Total genomic DNA was extracted by TIANamp Blood DNA kit (Tiangen Biotech, Beijing, China) following the manufacturer's instructions. The concentration of DNA was determined using the GeneQuant pro RNA/DNA Calculator (GE Healthcare, Pittsburgh, PA, USA) and the integrity of DNA was determined by electrophoresis.

HpaII-McrBC PCR assay. Human genomic DNA $(0.5 \mu \mathrm{g})$ was digested with 15 units HpaII or HhaI (Promega Corp.,
Madison, WI, USA) or 100 units McrBC (New England Biolabs, Ipswich, MA, USA) overnight at $37^{\circ} \mathrm{C}$ in $50 \mu 1$ of the buffers recommended by the suppliers. Subsequently, the enzymes were inactivated at $65^{\circ} \mathrm{C}$ for $20 \mathrm{~min}$ and the levels of digested DNA were determined by electrophoresis.

For PCR analysis, $0.5 \mu \mathrm{l}(5 \mathrm{ng})$ genomic DNA digested with each enzyme was used in a $10 \mu \mathrm{l}$ reaction mixture containing 0.25 units Ex-Taq DNA polymerase (Takara Bio Inc., Shiga, Japan), $4 \mathrm{nmol}$ dNTP (Takara Bio Inc.) and $10 \mathrm{nmol}$ each primer [Sangon Biotech (Shanghai) Co., Ltd., Shanghai, China] in $5 \mu 12$ X GC Buffer I or 2X GC Buffer II (Takara Bio Inc.). The thermal cycling parameters were recommend by Yamada et al (11). The amplified products were electrophoresed on a $2 \%$ agarose gel, stained with Goldview nucleic acid stain (SBS Genetech, Beijing, China) and visualized by the Molecular Imager Gel Doc XR+ System (Bio-Rad, Hercules, CA USA).

Bisulfite genomic sequencing. Human genomic DNA $(2 \mu \mathrm{g})$ from PBLs was treated with sodium bisulfite according to the standard procedure. One-tenth of the bisulfite-treated DNA was used for PCR in a $50 \mu \mathrm{l}$ reaction mixture, 10X PCR Buffer [100 mM Tris- $\mathrm{HCl}(\mathrm{pH} 8.8), 500 \mathrm{mM} \mathrm{KCl}, 15 \mathrm{mM} \mathrm{MgCl}_{2}$ and $0.8 \%$ (v/v) Nonidet P40], $10 \mathrm{nmol}$ each dNTP, $10 \mathrm{nmol}$ each primer and 4 units Ex-Taq DNA polymerase [all Sangon Biotech (Shanghai) Co., Ltd.]. The primer sequences are described in Table I. The amplified products were subsequently cloned into a pUC18-T vector [Sangon Biotech (Shanghai) Co., Ltd.] and sequenced using the Applied Biosystem 3730 DNA Analyzer (Life Technologies, Carlsbad, CA, USA). The results were further analyzed using the BDPC DNA methylation analysis platform (http://services.ibc.uni-stuttgart.de/BDPC/BISMA/).

Statistical analysis. Statistical analyses were performed using SPSS 10.0 software (SPSS Inc., Chicago, IL, USA). Comparisons between two groups were made using $\chi^{2}$ tests. $\mathrm{P}<0.05$ was considered to indicate a statistically significant difference.

\section{Results}

CGI sequence alignment data from the BGI YH database. There are a total of 149 CGIs in chromosome 21q, according to criteria outlined by Yamada et al (11). The alignment data from the BGI YH database showed that $87 \%$ of CGIs (130 of 149) were $>80 \%$ matched; $13.42 \%$ were a perfect match (20 of 149), $22.14 \%$ were $>95 \%$ matched (33 of 149), $21.48 \%$ were $>90 \%$ matched ( 32 of 149), $16.11 \%$ were $>85 \%$ matched ( 24 of 149) and $14.09 \%$ were $>85 \%$ matched (21 of 149). A match of $<80 \%$ accounted for $13 \%$ of total CGIs (19 of 149); $2.01 \%$ were $>75 \%$ matched ( 3 of 149 ), $8.05 \%$ were $>70 \%$ matched (12 of 149 ), $0.67 \%$ were $>65 \%$ matched ( 1 of 149 ), $1.34 \%$ were $>60 \%$ matched ( 2 of 149 ) and $0.67 \%$ were $>55 \%$ matched ( 1 of 149). The primers of the 148 CGIs were obtained from the experimental design of Yamada et al (11). The alignment data indicated that $98 \%$ (145 of 148) of the primers were a perfect match, two pairs of primers (CGI nos. 27 and 75) were a 50\% match and one pair of primers (CGI no. 90) was not a match. New primers for CGI nos. 27, 75 and 90 were designed using Primer 3 (v0.4.0; http://primer3.wi.mit.edu/) as follows: 


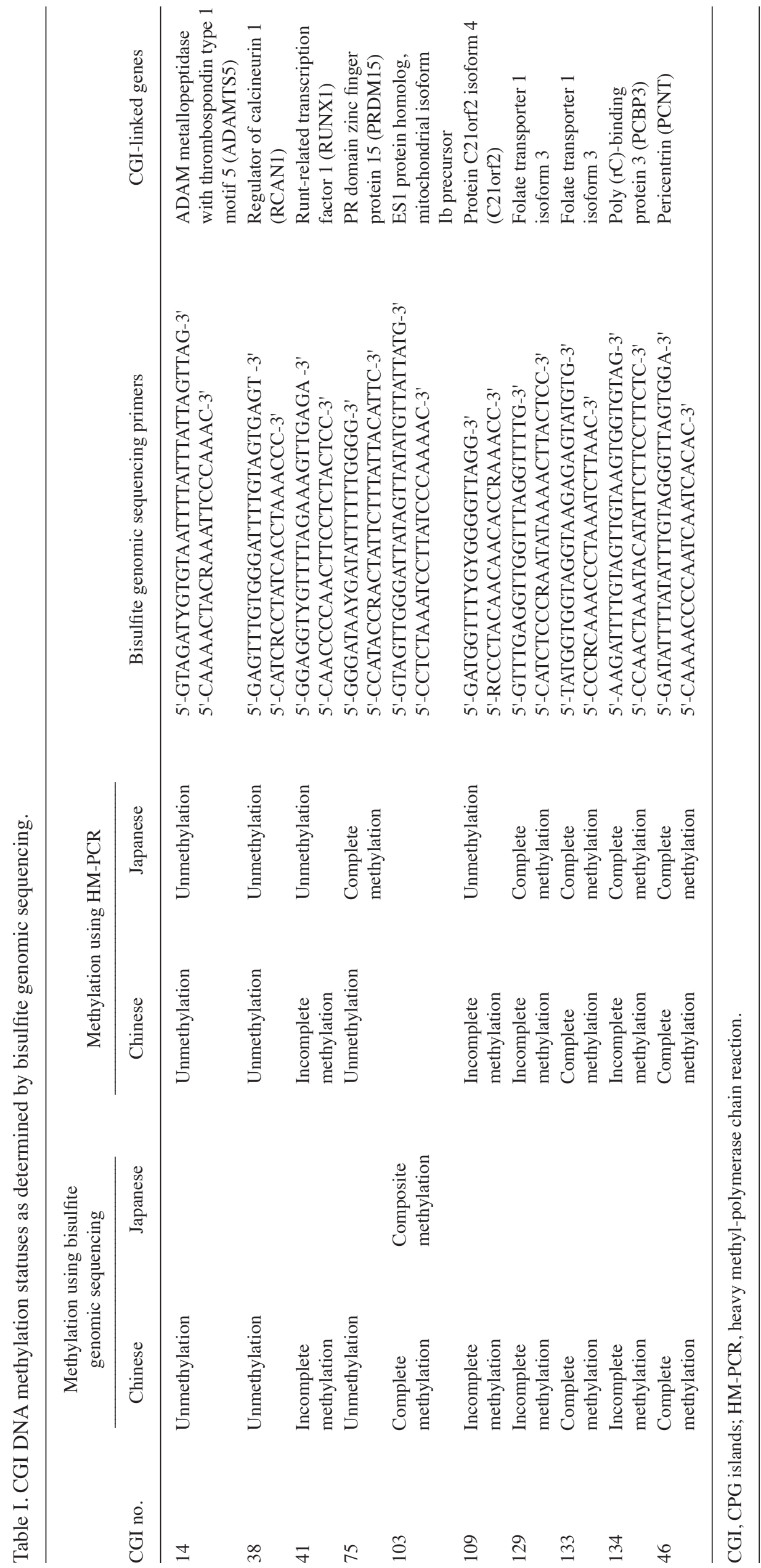




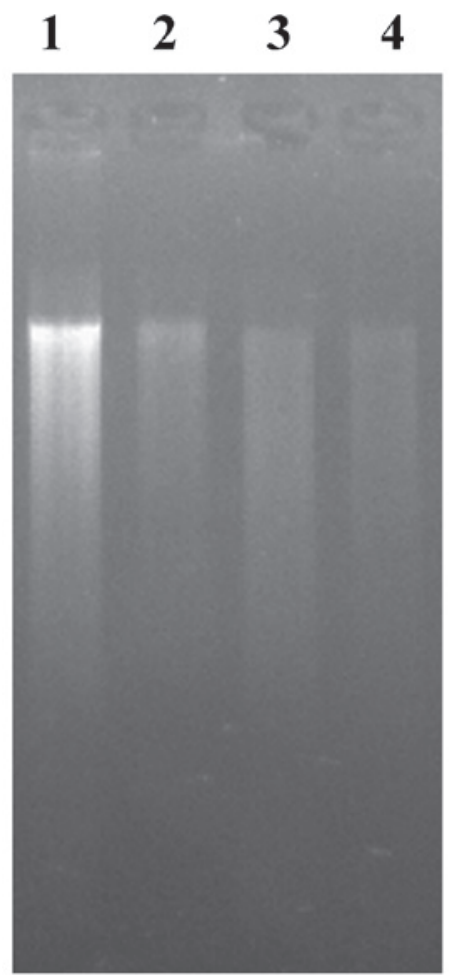

Figure 1. Human genomic DNA incubated with $M c r B C, H p a I I$ and HhaI enzymes. Lanes 1, human genomic DNA; 2, DNA incubated with $M c r B C$; 3, DNA incubated with HpaII; and 4, DNA incubated with HhaI.

Forward: 5'-CTCTCACCGCCGCAAGTCGGTCGC-3' and reverse: 5'-CGTTGTTGGGGAACTTTTACTGTG-3' for CGI no. 27; forward: 5'-CAGCTCAAGAATGCACTGCATTT-3' and reverse: 5'-AGTCAAAACCCGGCTGGATTTCC-3' for CGI no. 75; and forward: 5'-GTATGTGCCACCAAA TGATTATTCCT-3' and reverse: 5'-ACTCACTCTCCTAAC TTGAAGTTTTC-3' for CGI no. 90.

HpaII-McrBC PCR assay to evaluate 21q CGI allelic methylation status. The electrophoresed images of genomic DNA and DNA products digested by McrBC, HpaII or HhaI enzymes, respectively, are shown in Fig. 1. When the genomic DNA were completely digested by the enzyme, the DNA products appeared as smeared bands in gel electrophoresis. Using the heavy methyl-polymerase chain reaction (HM-PCR) assay, results showed that there was almost no difference in the DNA methylation status of 21q CGIs among individuals with trisomy 21 and the control, as shown in Table II. A total of 148 CGIs in 21q were screened, including 102 null methylation, 26 complete methylation, 7 composite methylation and 13 incomplete methylation. However, 3 null methylation CGIs (nos. 12, 41 and 109), 2 complete methylation (nos. 129 and 134) and 1 composite methylation (no. 55) in Japanese patients were all incomplete methylation in Chinese patients. In addition, 1 incomplete methylation CGI (no. 68) and 1 complete methylation CGI (no. 75) in Japanese patients were also incomplete methylation in Chinese patients. Finally, 1 complete methylation CGIs (nos. 1 and 137) in Japanese patients were composite methylation in Chinese. In total, there were 10 CGIs that showed varying DNA methylation statuses among Japanese and Chinese patients, as presented in Fig. 2 and Table II.

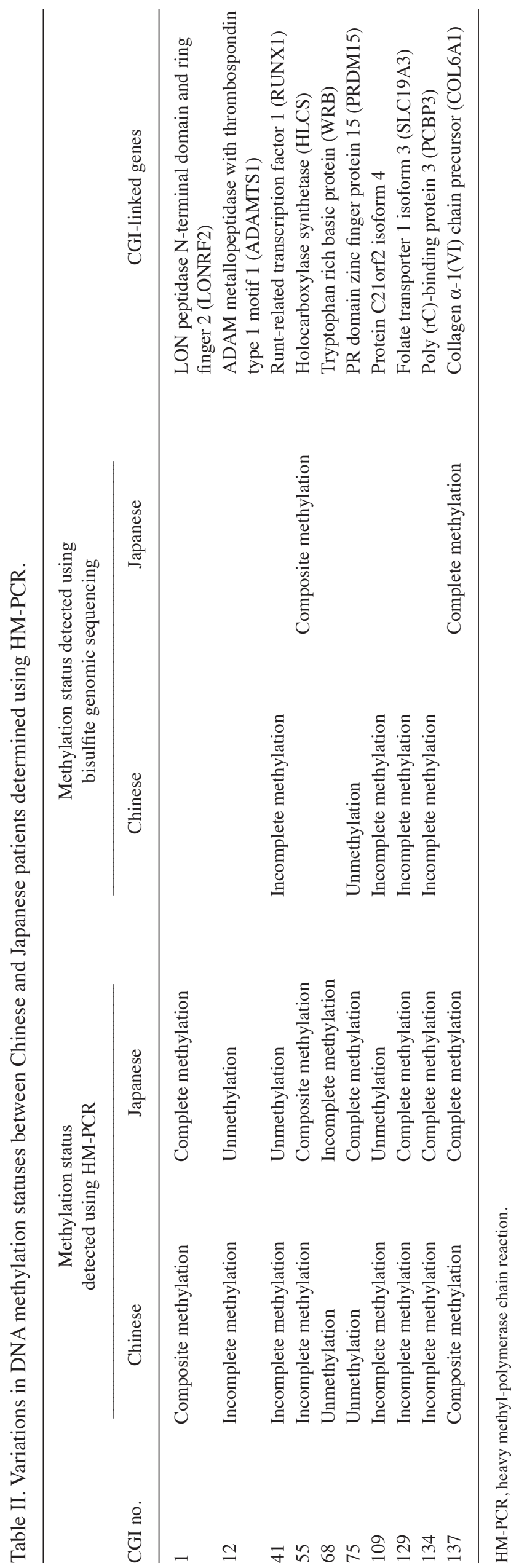



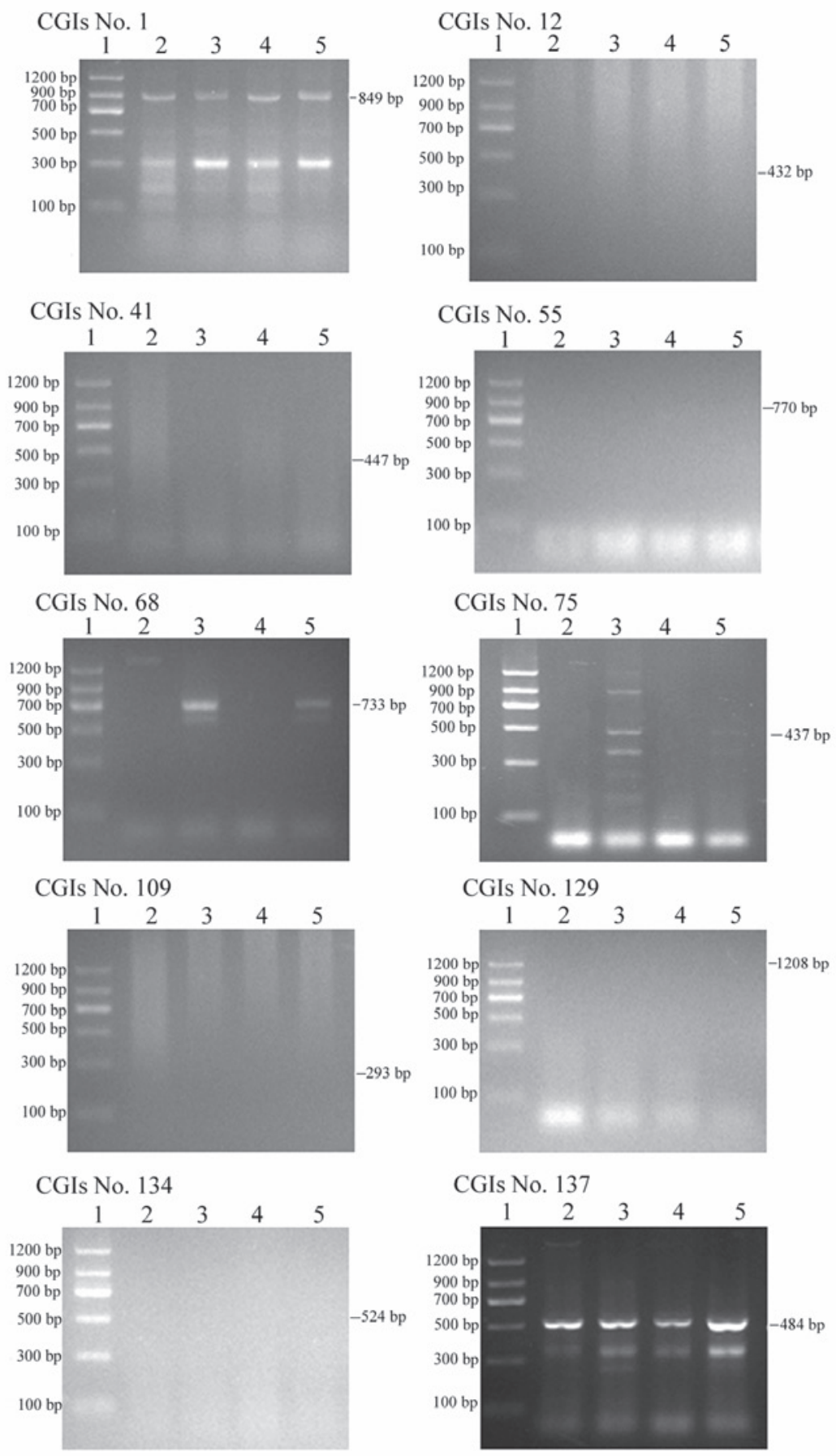

Figure 2. Representative agarose gel showing polymerase chain reaction (PCR) products of ten methylation CGIs on chromosome 21q. Lane 1, marker; 2, PCR products of HpaII digested DNA from a individual with Down's syndrome; 3, PCR products of McrBC digested DNA from a individual with Down's syndrome; 4, PCR products of HpaII digested DNA from the control; and 5, PCR products of McrBC digested DNA from the control. CGI, CpG island.

Bisulfite genomic sequencing to confirm allelic methylation status. Nine CGIs (nos. 14, 38, 41, 75, 109, 129, 133, 134 and 146) were selected to validate the HM-PCR assay data using bisulfite sequencing in trisomy 21 and the control, as shown in Table I. These validations were determined with bisulfite sequencing, which showed that CGI nos. 41, 109, 129 and 134 were incomplete methylation, CGI nos. 14, 38 and 75 were null methylation and CGI nos. 133 and 146 were complete methylation in Chinese patients, as shown in Fig. 3; these data validate the HM-PCR results. The methylation status of CGI no. 103 was detected by bisulfite sequencing, as it was short of the HpaII and HhaI recognition sites. The composite methylation CGI no. 103 in Japanese patients was complete methylation in Chinese patients, as shown in Table I and Fig. 3. Furthermore, the various methylation statuses of $\mathrm{CpG}$ dinucleotides in 5 CGIs between trisomy 21 and the control were found, including 5 sites in CGI no. 14, 6 sites in CGI no. 75, 14 sites in CGI no. 109, 1 site in CGI no. 134 and 3 sites in CGI no. 146. A total of $29 \mathrm{CpG}$ dinucleotides presented allele-specific DNA methylation, as shown in Fig. 3. 


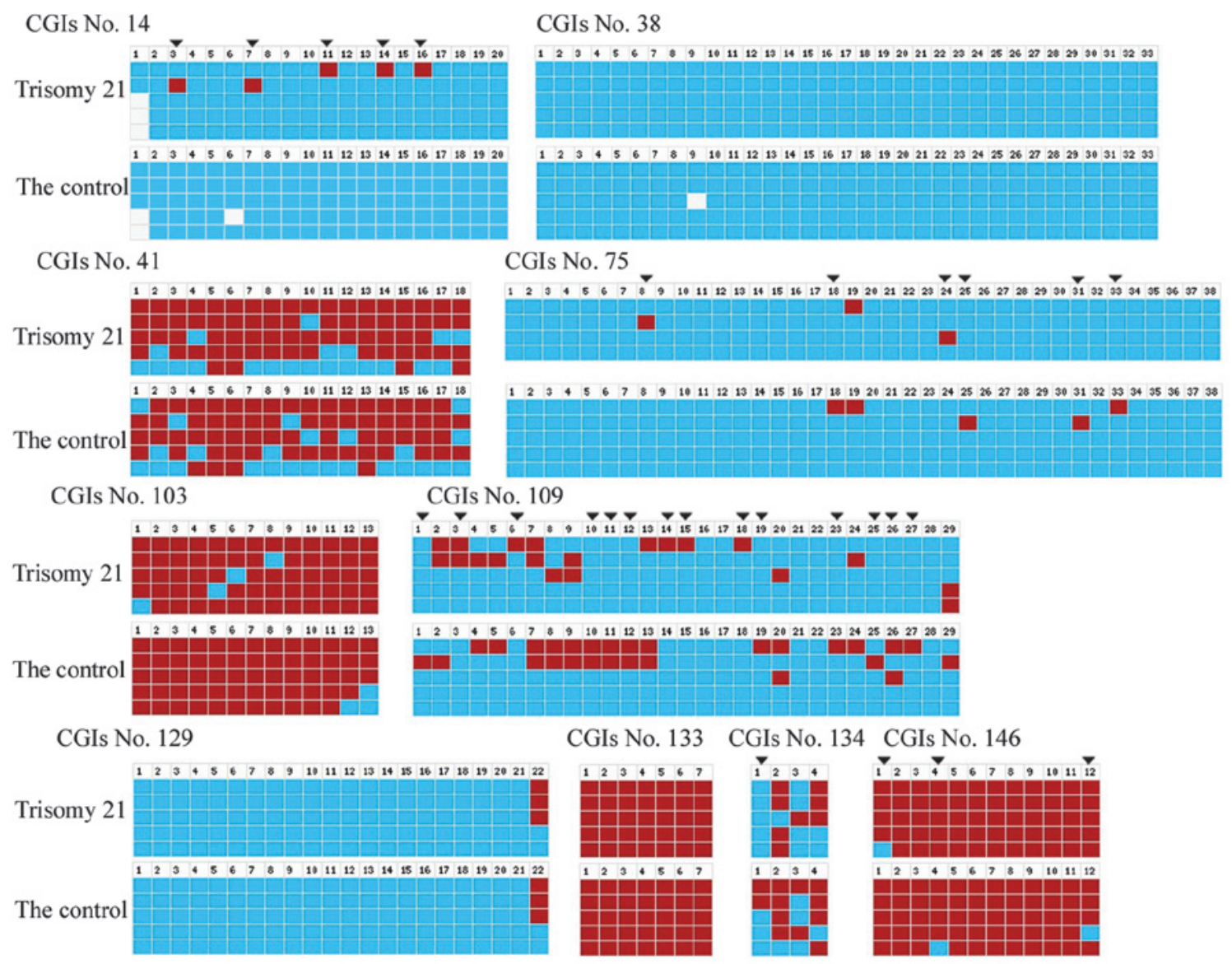

Figure 3. Methylation status of ten CGIs on chromosome 21q as determined by bisulfite genomic sequencing, defined as methylated (red), unmethylated (blue) and unknown (white). The different methylation statuses in $\mathrm{CpG}$ dinucleotide sites between trisomy 21 and the controls are indicated by downward arrows. Image depicts the various methylation CpG dinucleotide sites in CGI no. 14 (nos. 3, 7, 11, 14 and 16), 75 (nos. 8, 18, 24, 25, 31 and 33), 109 (nos. 1, 3, 6, 10, 11, 12, 14, 15, 18, 19, 23, 25, 26 and 27), 134 (no. 1) and 146 (no. 1, 4 and 12). CGI, CpG island.

\section{Discussion}

Individuals with DS have an additional chromosome 21, which is associated with the gene-dosage effect and a wide spectrum of phenotypic consequences, including life-threatening complications, clinically significant alteration of life course (e.g., mental retardation) and dysmorphic physical features (12). The mechanisms of gene regulation, include function of conserved nongenic regions, microRNA activities, RNA editing and DNA methylation. DS with a CHD is associated with a global hypomethylation status (13). DNA methylation is a possible mechanism of gene expression alteration, which may contribute to various abnormalities. Chango et al (14) used a combination of methylation-sensitive arbitrarily primed PCR and quantitation of DNA fragments to find six fragments that were hypermethylated in PBLs from eight individuals with DS, compared with eight normal controls. Kerkel et al (10) observed that 8 genes had different methylation status between the DS patients and normal controls. One of the 8 genes is named SUMO3 and is located on chromosome 21. The current observations are consistent with this data. There were differences in the DNA methylation status of $\mathrm{CpG}$ dinucleotide sites in 21q CGIs (nos. 14, 75, 109, 134 and 146) among individuals with trisomy 21 and the control. Molecular analysis reveals that the 21q22.1-q22.3 region, also known as the DS critical region (DSCR), appears to contain the gene or genes responsible for the CHD observed in DS (15-17). Altered DNA methylation in 21q may be constitutively silenced overexpressed genes in DS (10). Noteworthy gene candidates for specific dysfunctions in DS are already emerging from these research data. In the present study, CGI no. 75 was linked to PRDM15, which is a candidate gene for a particular phenotype of DS or bipolar affective disorder (18). CGI no. 14 was associated with ADAMTS5, which is a protease involved in regulating aggrecanase activity in cartilage; deletion of active ADAMTS5 prevents cartilage degradation $(19,20)$. CGI no. 109 was associated with C2lorf2, which is involved in the regulation of cell morphology and cytoskeletal organization (21). CGI no. 134 was associated with $P C B P 3$, which is associated with frontotemporal dementia and hypothesized to participate in mRNA metabolic processes $(22,23)$. CGI no. 146 was associated with $P C N T$, which may be important in preventing premature centrosome splitting during interphase by inhibition of NEK2 kinase activity at the centrosome (24). The methylation alteration may be associated with the DS phenotype, as insights from investigating DS as a model system have shed light on potential epigenetic biomarkers for noninvasive prenatal diagnosis in the general population.

The fetal-specific DNA methylation ratio permits noninvasive prenatal diagnosis of trisomy 21 by analyzing fetal-specific DMRs in free fetal DNA of the maternal circulation during pregnancy (25-28). A few differentially methylated sequences 
are fetal DNA methylation markers, including $H L C S$ and DSCR4 located at chromosome 21, RASSF1A located at chromosome 3 and $Z F Y$ located at chromosome Y (29-31). However, these markers were not specific for individuals with DS. In the present study, methylation alteration in DS may represent a specific epigenetic biomarker for future prenatal diagnosis; however, DNA methylation must be further verified in fetuses with DS.

DNA methylation in DS individuals did not change significantly in comparison with the controls (14). In the present study, there was no significant difference in 21q CGIs in Chinese patients, as determined using HM-PCR; this result was in accordance with observations from Kerkel et al (10) of no significant difference between normal and DS samples. While the different methylation statuses of $\mathrm{CpG}$ dinucleotide sites between the normal and DS do not cause the difference in the CGIs' methylation status as screened by HM PCR assays, because CGIs comprise numerous $\mathrm{CpG}$ dinucleotide sites only a few altered methylation levels are likely to not affect HM PCR results (11). Varying DNA methylation statuses of CGIs in 21q existed among Japanese and Chinese patients. According to the results of alignment in the BGI YH database, data showed that $87 \%$ of CGIs (130 of 149) were $>80 \%$ matched and 149 CGIs were feasible for the analysis of Chinese DNA sequences in the HM-PCR assay (32). In total, 149 CGIs in 21q were screened, including 102 null methylation, 26 complete methylation, 7 composite methylation and 13 incomplete methylation. There were 11 DNA methylation statuses of CGIs, no. 1, 12, 41, 55, 68, 75, 103, 109, 129, 134 and 137, among Japanese and Chinese patients. The racial disparities in DNA methylation patterns of differing ethnic groups implicate the probable role of molecular markers in determining an individual's susceptibility to disease. Racial disparities in DNA methylation patterns have been found in prostate cancer, endometrial carcinoma, breast cancer and laryngeal cancer and, in addition, are associated with racial difference in the cancer prognosis and survival rate (33-37). CGI no. 12 is associated with ADAMTS1, which is a protease involved in extracellular matrix proteolysis and antiangiogenesis and is involved in ischemia-induced retinal neovascularization, overexpressed in neurodegenerative disorders and downregulated in breast carcinomas (38-41). CGI no. 41 is associated with $R U N X 1$, which is linked to a poor outcome of acute lymphoblastic leukemia and susceptibility to autoimmune disease; emergence of the RUNX1 mutations was detected in advanced chronic myelogenous leukemias with acquired trisomy 21 (42-46). CGI no. 55 is associated with $H L C S$ and a lack of HLCS may cause multiple carboxylase deficiency (47). CGI no. 68 is associated with $W R B$, which is a conserved tryptophan-rich motif in the membrane-proximal region of the HIV-1 gp41 ectodomain and is important for Env-mediated fusion and virus infectivity $(46,48)$. CGI no. 129 is associated with $S L C 19 A 1$, a transporter for the intake of folate (49). CGI no. 137 is associated with COL6A1, depletion of which is a cause of Bethlem myopathy and Ullrich congenital muscular dystrophy (50).

In conclusion, the different DNA methylation status of 21q CGIs status between Chinese and Japanese individuals, and the same DNA methylation status detected by HM-PCR between Chinese individuals with DS and the control indicates that DNA methylation is likely to be an epigenetic marker for distinguishing ethnicities. The different DNA methylation status of $\mathrm{CpG}$ dinucleotides between individuals with DS and the control may contribute to the DS complex phenotypes and be a potential epigenetic marker for diagnosing trisomy 21 .

\section{Acknowledgements}

The authors would like to thank Dr Lin Zou and Dr Jing Yang (Center for Clinical Molecular Medicine, Children's Hospital of Chongqing Medical University) for case collection. The present study was supported by grants from the Chongqing Key Laboratory of Birth Defects and Reproductive Health (grant no. 0901) and the Natural Science Foundation Project of CQ CSTC (grant no. 2009BA5082).

\section{References}

1. Huether CA, Gummere GR, Hook EB, et al: Down's syndrome: percentage reporting on birth certificates and single year maternal age risk rates for Ohio 1970-79: comparison with upstate New York data. Am J Public Health 71: 1367-1372, 1981.

2. Karlsson B, Gustafsson J, Hedov G, Ivarsson SA and Anneren G: Thyroid dysfunction in Down's syndrome: relation to age and thyroid autoimmunity. Arch Dis Child 79: 242-245, 1998.

3. Nespoli L, Burgio GR, Ugazio AG and Maccario R: Immunological features of Down's syndrome: a review. J Intellect Disabil Res 37: 543-551, 1993.

4. Yang Q, Rasmussen SA and Friedman JM: Mortality associated with Down's syndrome in the USA from 1983 to 1997: a population-based study. Lancet 359: 1019-1025, 2002.

5. Caputo AR, Wagner RS, Reynolds DR, Guo SQ and Goel AK: Down syndrome. Clinical review of ocular features. Clin Pediatr (Phila) 28: 355-358, 1989.

6. Shott SR, Joseph A and Heithaus D: Hearing loss in children with Down syndrome. Int J Pediatr Otorhinolaryngol 61: 199-205, 2001.

7. Lejeune J, Turpin R and Gautier M: Mongolism; a chromosomal disease (trisomy). Bull Acad Natl Med 143: 256-265, 1959 (In French).

8. Daura-Oller E, Cabre M, Montero MA, Paternain JL and Romeu A: Specific gene hypomethylation and cancer: new insights into coding region feature trends. Bioinformation 3 : 340-343, 2009.

9. Smolarek I, Wyszko E, Barciszewska AM, et al: Global DNA methylation changes in blood of patients with essential hypertension. Med Sci Monit 16: CR149-CR155, 2010.

10. Kerkel K, Schupf N, Hatta K, et al: Altered DNA methylation in leukocytes with trisomy 21. PLoS Genet 6: e1001212, 2010.

11. Yamada Y, Watanabe H, Miura F, et al: A comprehensive analysis of allelic methylation status of $\mathrm{CpG}$ islands on human chromosome 21q. Genome Res 14: 247-266, 2004.

12. Patterson D: Genetic mechanisms involved in the phenotype of Down syndrome. Ment Retard Dev Disabil Res Rev 13: 199-206, 2007.

13. Obermann-Borst SA, van Driel LM, Helbing WA, et al: Congenital heart defects and biomarkers of methylation in children: a case-control study. Eur J Clin Invest 41: 143-150, 2011.

14. Chango A, Abdennebi-Najar L, Tessier F, et al: Quantitative methylation-sensitive arbitrarily primed PCR method to determine differential genomic DNA methylation in Down Syndrome. Biochem Biophys Res Commun 349: 492-496, 2006.

15. Laue L, Chan WY, Hsueh AJ, et al: Genetic heterogeneity of constitutively activating mutations of the human luteinizing hormone receptor in familial male-limited precocious puberty. Proc Natl Acad Sci USA 92: 1906-1910, 1995.

16. Arron JR, Winslow MM, Polleri A, et al: NFAT dysregulation by increased dosage of DSCR1 and DYRK1A on chromosome 21. Nature 441: 595-600, 2006

17. Ronan A, Fagan K, Christie L, et al: Familial 4.3 Mb duplication of 21q22 sheds new light on the Down syndrome critical region. J Med Genet 44: 448-451, 2007.

18. Shibuya K, Kudoh J, Okui M and Shimizu N: Identification of a novel zinc finger protein gene (ZNF298) in the GAP2 of human chromosome 21q. Biochem Biophys Res Commun 332: 557-568, 2005. 
19. Vankemmelbeke MN, Holen I, Wilson AG, et al: Expression and activity of ADAMTS-5 in synovium. Eur J Biochem 268: 1259-1268, 2001.

20. Glasson SS, Askew R, Sheppard B, et al: Deletion of active ADAMTS5 prevents cartilage degradation in a murine model of osteoarthritis. Nature 434: 644-648, 2005.

21. Bai SW, Herrera-Abreu MT, Rohn JL, et al: Identification and characterization of a set of conserved and new regulators of cytoskeletal organization, cell morphology and migration. BMC Biol 9: 54, 2011.

22. Kiledjian M, Wang X and Liebhaber SA: Identification of two KH domain proteins in the alpha-globin mRNP stability complex. EMBO J 14: 4357-4364, 1995.

23. Wang Y, Gao L, Tse SW and Andreadis A: Heterogeneous nuclear ribonucleoprotein E3 modestly activates splicing of tau exon 10 via its proximal downstream intron, a hotspot for frontotemporal dementia mutations. Gene 451: 23-31, 2010.

24. Matsuo K, Nishimura T, Hayakawa A, Ono Y and Takahashi M: Involvement of a centrosomal protein kendrin in the maintenance of centrosome cohesion by modulating Nek2A kinase activity. Biochem Biophys Res Commun 398: 217-223, 2010.

25. Papageorgiou EA, Karagrigoriou A, TsalikiE, et al: Fetal-specific DNA methylation ratio permits noninvasive prenatal diagnosis of trisomy 21. Nat Med 17: 510-513, 2011.

26. Papageorgiou EA, Fiegler H, Rakyan V, et al: Sites of differential DNA methylation between placenta and peripheral blood: molecular markers for noninvasive prenatal diagnosis of aneuploidies. Am J Pathol 174: 1609-1618, 2009.

27. Old RW, Crea F, Puszyk W and Hultén MA: Candidate epigenetic biomarkers for non-invasive prenatal diagnosis of Down syndrome. Reprod Biomed Online 15: 227-235, 2007.

28. Chan KC, Ding C, Gerovassili A, et al: Hypermethylated RASSF1A in maternal plasma: A universal fetal DNA marker that improves the reliability of noninvasive prenatal diagnosis. Clin Chem 52: 2211-2218, 2006

29. Chim SS, Jin S, Lee TY, et al: Systematic search for placental DNA-methylation markers on chromosome 21: toward a maternal plasma-based epigenetic test for fetal trisomy 21. Clin Chem 54: 500-511, 2008

30. Du Y, Zhang J, Wang H, et al: Hypomethylated DSCR4 is a placenta-derived epigenetic marker for trisomy 21. Prenat Diagn 31: 207-214, 2011.

31. Tong YK, Jin S, Chiu RW, et al: Noninvasive prenatal detection of trisomy 21 by an epigenetic-genetic chromosome-dosage approach. Clin Chem 56: 90-98, 2010.

32. Wang J, Wang W, Li R, et al: The diploid genome sequence of an Asian individual. Nature 456: 60-65, 2008 .

33. Woodson K, Hayes R, Wideroff L, Villaruz L and Tangrea J: Hypermethylation of GSTP1, CD44, and E-cadherin genes in prostate cancer among US Blacks and Whites. Prostate 55: 199-205, 2003

34. Woodson K, Hanson J and Tangrea J: A survey of gene-specific methylation in human prostate cancer among black and white men. Cancer Lett 205: 181-188, 2004.

35. Adkins RM, Krushkal J, Tylavsky FA and Thomas F: Racial differences in gene-specific DNA methylation levels are present at birth. Birth Defects Res A Clin Mol Teratol 91: 728-736, 2011
36. Nielsen DA, Hamon S, Yuferov V, et al: Ethnic diversity of DNA methylation in the OPRM1 promoter region in lymphocytes of heroin addicts. Hum Genet 127: 639-649, 2010.

37. Dumitrescu RG, Marian C, Krishnan SS, et al: Familial and racial determinants of tumour suppressor genes promoter hypermethylation in breast tissues from healthy women. J Cell Mol Med 14: 1468-1475, 2010.

38. Bevitt DJ, Mohamed J, Catterall JB, et al: Expression of ADAMTS metalloproteinases in the retinal pigment epithelium derived cell line ARPE-19: transcriptional regulation by TNFalpha. Biochim Biophys Acta 1626: 83-91, 2003.

39. Porter S, Scott SD, Sassoon EM, et al: Dysregulated expression of adamalysin-thrombospondin genes in human breast carcinoma. Clin Cancer Res 10: 2429-2440, 2004.

40. Xu Z, Yu Y and Duh EJ: Vascular endothelial growth factor upregulates expression of ADAMTS1 in endothelial cells through protein kinase C signaling. Invest Ophthalmol Vis Sci 47: 4059-4066, 2006 .

41. Miguel RF, Pollak A and Lubec G: Metalloproteinase ADAMTS-1 but not ADAMTS-5 is manifold overexpressed in neurodegenerative disorders as Down syndrome, Alzheimer's and Pick's disease. Brain Res Mol Brain Res 133: 1-5, 2005.

42. Helms C, Cao L, Krueger JG, et al: A putative RUNX1 binding site variant between SLC9A3R1 and NAT9 is associated with susceptibility to psoriasis. Nat Genet 35: 349-356, 2003.

43. Liu S, Shen T, Huynh L, et al: Interplay of RUNX1/MTG8 and DNA methyltransferase 1 in acute myeloid leukemia. Cancer Res 65: 1277-1284, 2005.

44. Roche-Lestienne C, Deluche L, Corm S, et al: RUNX1 DNA-binding mutations and RUNX1-PRDM16 cryptic fusions in BCR-ABL ${ }^{+}$leukemias are frequently associated with secondary trisomy 21 and may contribute to clonal evolution and imatinib resistance. Blood 111: 3735-3741, 2008.

45. Strefford JC, van Delft FW, Robinson HM, et al: Complex genomic alterations and gene expression in acute lymphoblastic leukemia with intrachromosomal amplification of chromosome 21. Proc Natl Acad Sci USA 103: 8167-8172, 2006.

46. Zhang Y, Strissel P, Strick R, et al: Genomic DNA breakpoints in AML1/RUNX1 and ETO cluster with topoisomerase II DNA cleavage and DNase I hypersensitive sites in $\mathrm{t}(8 ; 21)$ leukemia. Proc Natl Acad Sci USA 99: 3070-3075, 2002.

47. Tang NL, Hui J, Yong CK, et al: A genomic approach to mutation analysis of holocarboxylase synthetase gene in three Chinese patients with late-onset holocarboxylase synthetase deficiency. Clin Biochem 36: 145-149, 2003.

48. Salzwedel K, West JT and Hunter E: A conserved tryptophan-rich motif in the membrane-proximal region of the human immunodeficiency virus type $1 \mathrm{gp} 41$ ectodomain is important for Env-mediated fusion and virus infectivity. J Virol 73: 2469-2480, 1999.

49. Börjel AK, Yngve A, Sjöström M and Nilsson TK: Novel mutations in the 5'-UTR of the FOLR1 gene. Clin Chem Lab Med 44: 161-167, 2006.

50. Lampe AK, Dunn DM, von Niederhausern AC, et al: Automated genomic sequence analysis of the three collagen VI genes: applications to Ullrich congenital muscular dystrophy and Bethlem myopathy. J Med Genet 42: 108-120, 2005. 